Military Technical College, Kobry El-Kobbah, Cairo, Egypt

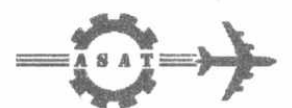

$9^{\text {th }}$ International Conference On Aerospace Sciences \& Aviation Technology

\title{
RESTRICTIVE PADE` APPROXIMATION FOR SOLVING SINGULARLY PERTURBED INITIAL BOUNDARY VALUE PROBLEM FOR HYPERBOLIC PARTIAL DIFFERENTIAL EQUATION
}

\author{
Hassan N. A. Ismail" and Adel Y. H. Elmekkawy"
}

\begin{abstract}
This paper is concerned with the numerical treatment of the first-order hyperbolic partial differential equation by small parameter with the time derivative term. This problem is reduced to stiff system of $O D E_{s}$ in time. The resulting system is solved by the restrictive Pade approximation. The stability condition and the error upper bound are introduced. The numerical results are given and the considered method gives better results compared with the classical advantages of the considered method compared with the classical Pade' approximation.
\end{abstract}

\section{KEY WORDS}

Pade` approximation, Restrictive Pade`approximation, finite difference and hyperbolic partial differential equations.

\footnotetext{
Benha Higher Institute of Technology, Benha, Egytp.

Dept. of Math. Faculty of Education, Ain Shams University
} 


\section{Introduction}

Consider the singularly perturbed first-order hyperbolic partial differential equation:

$$
\delta \frac{\partial u}{\partial t}+a \frac{\partial u}{\partial x}=f(x, t) ; \quad x>0, t \geq 0
$$

where $\delta>0$ is small, $a$ is real positive constants and $f(x, t)$ is given continuous function satisfies the initial and boundary conditions:

$$
\left.\begin{array}{ll}
u(x, 0)=u_{0}(x), & 0 \leq x \leq 1 \\
u(0, t)=g_{0}(t), & u(1, t)=g_{1}(t), t \geq 0
\end{array}\right\} .
$$

Using the central finite difference approximation for $u_{x}$ as

$$
u(i h, t)=\frac{1}{2 h}[(u(i+1) h, t)-(u(i-1) h, t)] ; \quad i=1(1) N-1 .
$$

The resulting semi-discrete approximation $U(i h, t)$ to $u(x, t)$ of equation (1) satisfies

$$
\frac{d U(i h, t)}{d t}=\frac{a}{2 \delta h}[U((i-1) h, t)-U((i+1) h, t)]+\frac{1}{\delta} f\left(x_{i}, t\right) ; \quad 1 \leq i \leq N-1, t \geq 0
$$

where $U(i h, 0)=u_{0}(i h), \quad U(0, t)=g_{0}(t)$, and $U(N h, t)=g_{1}(t), t \geq 0$, it can be written in matrix form as:

$$
\frac{d \underline{U}(t)}{d t}=A \underline{U}(t)+\underline{F}(t),
$$

where

$$
\underline{U}(t)=\left(U_{1}(t), U_{2}(t), \cdots, U_{N-1}(t)\right)^{T}
$$

$U_{i}(t)=U(i h, t), f_{i}(t)=f(i h, t)$ and

$$
A=\left[\begin{array}{cccccc}
0 & -1 & & & & \\
1 & 0 & -1 & & & \\
& \ddots & \ddots & \ddots & & \\
& & \ddots & \ddots & \ddots & \\
& & & 1 & 0 & -1 \\
& & & & 1 & 0
\end{array}\right]_{(N-1)(N-1)}
$$

The solution of this system of ordinary differential equations (4) as done in [10] and [11] take the form:

$$
\underline{U}(t)=\exp (t A) \underline{U}(0)+[-I+\exp (t A)] A^{-1} \underline{F} .
$$

Or equivalently

$$
\underline{U}(t+\Delta t)=-A^{-1} \underline{F}+\exp (\Delta t A)\left[\underline{U}(t)+A^{-1} F\right] .
$$

If we approximate $\exp (\Delta t A)$ using Pade approximation [M/N](PA[M/N), then we can write equations (6) for any time step $j$ as:

$$
\underline{U}^{j+1}=-A^{-1} \underline{F}+P A_{\text {exp }}^{(\alpha+1)}\left[U^{j}+A^{-1} \underline{F}\right], \quad \Delta t=k
$$

In the following section we define an implicit method for solving singularly perturbed initial boundary value problem for hyperbolic partial differential equation produced very high accuracy compared with the other classical methods. We use 
the restrictive Pade' approximation as done in [1] and [2] to approximate the exponential matrix.

\section{Restrictive Pade`Approximation (RPA)} form

The restrictive Pade approximation can be written as done in [1] in the

$$
R P A[M+\alpha / N]_{f(x)}(x)=\frac{\sum_{i=0}^{M} a_{i} x^{i}+\sum_{i=1}^{\alpha} \varepsilon_{i} x^{M+i}}{1+\sum_{i=1}^{N} b_{i} x^{i}}
$$

where $\alpha$ is a positive integer dose not exceed the degree of the denominator $N$, i.e. $\quad \alpha=1(1) \mathrm{N}$, such that

$$
f(x)-R P A[M+\alpha / N]_{f(x)}(x)=o\left(x^{M+N+1}\right) .
$$

Let $f(x)$ have a Maclaurin series $f(x)=\sum_{i=0}^{\infty} c_{i} x^{i}$, then from equations (8) and (9) we have

$$
\left(\sum_{i=0}^{\infty} c_{i} x^{\prime}\right)\left(1+\sum_{i=1}^{N} b_{i} x^{i}\right)-\left(\sum_{i=0}^{M} a_{i} x^{i}\right)-\left(\sum_{i=1}^{\alpha} \varepsilon_{i} x^{i+M}\right)=o\left(x^{M+N+1}\right) .
$$

The vanishing of the first $(M+N+1)$ powers of $x$ on the left hand side of (10) implies a system of $(M+N+1)$ equations.

$$
\left.\begin{array}{ll}
a_{r}=c_{r}+\sum_{i=1}^{r} c_{r-i} b_{i}, & r=0(1) M, \\
c_{M+N-s}+\sum_{i=1}^{N} c_{M+N-i-s} b_{i}=\varepsilon_{N-s,}, & \left(b_{i}=0 \text { if } i>M\right) \\
s=0(1) N-1, \\
\left(c_{i}=0 \text { if } i<0\right)
\end{array}\right\}
$$

Hence we can determine the coefficient, $a_{i}$ and $b_{i}$ as a function of $a_{1} i=1(1) \alpha$, where the parameters $\varepsilon_{1}$ are to be determined, such that

$$
f\left(x_{i}\right)=R P A[M+\alpha / N]_{f(x)}\left(x_{i}\right), \quad i=1(1) \alpha .
$$

It means that the considered approximation is exact at $(\alpha+1)$ points.

Consider the function $\quad f(x)=\left(\frac{1+0.5 x+0.25 x^{2}}{1+5 x}\right)^{0.5}$,

its Pade' approximation and restrictive Pade' approximation takes the forms:

$$
\begin{aligned}
& P A[2 / 1]_{f(x)}(x)=\frac{1+1.9311 x-0.563724 x^{2}}{1+4.1811 x} \\
& R P A[2 / 1]_{f(x)}(x)=\frac{1+1.73134 x-0.114257 x^{2}}{1+3.98134 x} \text { where } \alpha=1 \text { and } x_{\alpha}=0.6
\end{aligned}
$$


3. Restrictive Pade Approximation (RPA) for Solving Singularly Perturbed Hyperbolic Partial Differential Equation

The restrictive Pade' approximation of order [0/1] and [1/1] of the exponential matrix $\exp (k A)$ as done in [3] can take the forms:

i-) $R P A[0 / 1]_{\exp (k A)}=(I+(\varepsilon-k) A)^{-1}$

using equation (13) to approximate the $\exp (k A)$ in equation (6), which can the form:

or

$$
\underline{U}^{j+1}=-A^{-1} \underline{F}+(I+(\varepsilon-k) A)^{-1}\left[\underline{U}^{j}+A^{-1} \underline{F}\right], \quad \Delta t=k,
$$

$$
(I+(\varepsilon-k) A) \underline{U}^{j+1}=\underline{U}^{j}-(\varepsilon-k) \underline{F} .
$$

which can take the equivalent scalar form:

$$
\left(\frac{(\varepsilon-k) a}{2 \delta h}\right) u_{i-1, j+1}+u_{i, j+1}-\left(\frac{(\varepsilon-k) a}{2 \delta h}\right) u_{i+1, j+1}=u_{i, j}-\left(\frac{\varepsilon-k}{\delta}\right) f\left(x_{i}, t\right) .
$$

Similarly the restrictive Pade' approximation of order [1/1] of the exponential matrix $\exp (k A)$ as done in [3] can take the form:

ii-) $R P A[1 / 1]_{\exp (k 4)}=\left(I+\left(\varepsilon-\frac{1}{2} k\right) A\right)^{-1}\left(I+\left(\varepsilon+\frac{1}{2} k\right) A\right)$

using equation (13) to approximate the $\exp (k A)$ in equation (6), which can the form:

$$
\underline{U}^{j+1}=-A^{-1} \underline{F}+\left(I+\left(\varepsilon-\frac{1}{2} k\right) A\right)^{-1}\left(I+\left(\varepsilon+\frac{1}{2} k\right) A\right)\left[\underline{U}^{j}+A^{-1} \underline{F}\right]
$$

or

$$
\left(I+\left(\varepsilon-\frac{1}{2} k\right) A\right) \underline{U}^{j+1}=\left(I+\left(\varepsilon+\frac{1}{2} k\right) A\right) \underline{U}^{j}+k \underline{F} .
$$

It can take the equivalent scalar form:

$$
\begin{aligned}
\left(\frac{(\varepsilon-0.5 k) a}{2 \delta h}\right) u_{i-1, j+1}+u_{i, j+1}-\left(\frac{(\varepsilon-0.5 k) a}{2 \delta h}\right) u_{i+1, j+1} \\
=\left(\frac{(\varepsilon+0.5 k) a}{2 \delta h}\right) u_{i-1, j}+u_{i, j}-\left(\frac{(\varepsilon+0.5 k) a}{2 \delta h}\right) u_{i+1, j}+\left(\frac{k}{\delta}\right) f\left(x_{i}, t\right) .
\end{aligned}
$$

Putting $\varepsilon=0$ in equations (16) and (20), we have the schemes arising of applying classical Pade' approximations $[0 / 1]$ and [1/1] respectively to the exponential matrix $\exp (\mathrm{kA})$ in equation (6). To determine the restrictive parameters $\varepsilon$, we must have the exact solution or a highly accurate numerical solution at the first level. 


\section{The Stability Analysis}

By using Von-Neumann stability analysis method we have, the amplification factors $G_{1}$ and $G_{2}$ for the difference equations (16) and (20) respectively are:

$$
G_{1}=\frac{1}{1-I\left(\frac{(\varepsilon-k) a}{\delta h} \sin \theta\right)}, \quad G_{2}=\frac{1-I\left(\frac{(\varepsilon+0.5 k) a}{\delta h} \sin \theta\right)}{1-I\left(\frac{(\varepsilon-0.5 k) a}{\delta h} \sin \theta\right)}, I=\sqrt{-1}
$$

i.e. $\left|G_{1}\right|<1, \forall \varepsilon$ and $\left|G_{2}\right|<1, \forall \varepsilon<0$ consequently the considered methods

(16) and (20) are unconditionally stable.

\section{The Local Truncation Error Upper Bound}

\section{i-) For the RPA[0/1]}

Using Taylor expansion, we can obtained the local truncation error of the difference equation (16) as done in [5],[6],[7],[8] and [9] as:

$T_{i, j}=\frac{(\varepsilon-k) a}{\delta h} \sum_{n=0}^{\infty}\left(\frac{h^{2 n+1}}{(2 n+1) !} \frac{\partial^{2 n+1} u}{\partial x^{2 n+1}}\right)_{(i, j)}+\sum_{n=1}^{\infty}\left(\frac{k^{n}}{n !} \frac{\partial^{n} u}{\partial t^{n}}\right)_{(i, j)}-\frac{(\varepsilon-k) a}{\delta h} \sum_{m=0}^{\infty} \frac{h^{2 m+1}}{(2 m+1) !} \sum_{n=1}^{\infty}\left(\frac{k^{n}}{n !} \frac{\partial^{2 m+n+1} u}{\partial x^{2 m+1} \partial t^{n}}\right)_{(i, j)}$

Then, if there exists a positive real numbers $M_{1}, M_{2}, M_{3}$ for all sufficiently large positive integer $n$ such that

$$
\left|\frac{\partial^{n} u}{\partial x^{n}}\right| \leq M_{1},\left|\frac{\partial^{n} u}{\partial t^{n}}\right| \leq M_{2},\left|\frac{\partial^{m+n} u}{\partial x^{m} \partial t^{n}}\right| \leq M_{3} \quad \forall n .
$$

Let $\quad M=\max \left\{M_{1}, M_{2}, M_{3}\right\}$

then, the local truncation error $T_{i j}$ of the difference equation (16) will have an upper bound as:

$$
\left|T_{i, j}\right| \leq M\left|e^{k}\left(1+\frac{(\varepsilon-k) a}{\delta h} \sinh h\right)-1\right| .
$$

\section{i- For the RPA[1/1]}

Also, in the same way using Taylor expansion, we can obtained the local truncation error of the difference equation (20) as:

$T_{i, j}=\left(r_{1}-r_{2}\right) \sum_{n=0}^{\infty}\left(\frac{h^{2 n+1}}{(2 n+1) !} \frac{\partial^{2 n+1} u}{\partial x^{2 n+1}}\right)_{(i, j)}+\sum_{n=1}^{\infty}\left(\frac{k^{n}}{n !} \frac{\partial^{n} u}{\partial t^{n}}\right)_{(i, j)}-r_{1} \sum_{m=0}^{\infty} \frac{h^{2 m+1}}{(2 m+1) !} \sum_{n=1}^{\infty}\left(\frac{k^{n}}{n !} \frac{\partial^{2 m+n+1} u}{\partial x^{2 m+1} \partial t^{n}}\right)_{(i, j)}$, 
where $r_{1}=\frac{(\varepsilon-0.5 k) a}{\delta h}, r_{2}=\frac{(\varepsilon+0.5 k) a}{\delta h}$, then if there exists a positive real numbers $M_{1}, M_{2}, M_{3}$ for all sufficiently large positive integer $n$ such that

$$
\left|\frac{\partial^{n} u}{\partial x^{n}}\right| \leq M_{1},\left|\frac{\partial^{n} u}{\partial t^{n}}\right| \leq M_{2},\left|\frac{\partial^{m+n} u}{\partial x^{m} \partial t^{n}}\right| \leq M_{3} \quad \forall n .
$$

and $M=\max \left\{M_{1}, M_{2}, M_{3}\right\}$,

then, the local truncation error $T_{i j}$ of the difference equation (20) will have an upper bound as:

$$
\left|T_{i, j}\right| \leq M\left|\left(r_{1} e^{k}-r_{2}\right) \sinh h+e^{k}-1\right|,
$$

\section{Numerical Results}

We present a numerical example to compare the considered methods (16) and (20) with the corresponding classical methods which arising of applying Pade' approximations $[0 / 1]$ and $[1 / 1]$ respectively to the exponential matrix exp ( $k$ A) in equation (6).

Consider the singularly perturbed hyperbolic partial differential equation

$$
\delta \frac{\partial u}{\partial t}+\frac{\partial u}{\partial x}=2 x-1,
$$

with the initial and boundary conditions

$$
u(x, 0)=\exp (-t / \delta), u(x, 0)=\exp (x)+x^{2}-x \text { and } u(x, 1)=\exp (x-1 / \delta)+x^{2}-x
$$

its exact solution is:

$$
u(x, t)=\exp (x-t / \delta)+x^{2}-x .
$$

We consider two cases:

i-) Case I : We apply our methods such that the exact solution is given at the first level to determine the restrictive parameters $\varepsilon_{i}$, and hence we use it for another levels for calculation. Tables (1) gives the absolute errors along $x=0.1,0.5,0.9$ where $h=0.1$ and $\delta=0.01, k=0.001$. Tables (2) gives the absolute errors along $x=0.1,0.5,0.9$ where $h=0.1$ and $\delta=0.001, k=0.0000 \%$.

ii-) Case II: In general the exact solution at the first level is unknown, and we use the classical method in the case of PA[1/1], to evaluate the solutions at the first time level by large number of very small space and time steps lengths $\mathrm{h}=0.01$ and $\mathrm{k}=1 \times 10^{-10}$, after 100 time step, $\mathrm{k}=1 \times 10^{-8}$ and we can choice space step $h=0.1$, hence we determine the restrictive parameters $\varepsilon$ i.e. we can use large space and time steps lengths $h$ and $k$ to evaluate the solution at another levels. Tables (3) give the absolute errors along $x=0.1,0.5,0.9$ where $h=0.1$ and $\delta=$ $0.0001, \mathrm{k}=1 \times 10^{-8}$. 


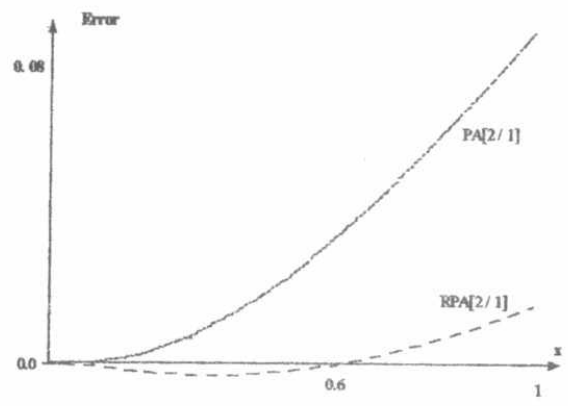

Fig (1)

Comparison of the errors between $\mathrm{PA}[2 / 1]$ and $\mathrm{RPA}[2 / 1]$

Table (1)

Comparison of the absolute errors (A.E.) between the considered methods RPA[1/1], RPA[0/1] and The classical methods PA[1/1], PA[O/1], for $h=0.1, k=1 \times 10^{-3}$ and $\delta=1 \times 10^{-2}$ for case $\mathrm{I}$.

\begin{tabular}{|c|c|c|c|c|c|}
\hline$x$ & $\begin{array}{c}\text { No. of } \\
\text { Steps }\end{array}$ & \multicolumn{2}{|c|}{$\begin{array}{c}\text { A. E. of The considered } \\
\text { methods }\end{array}$} & \multicolumn{2}{c|}{$\begin{array}{c}\text { A. E. of The classical } \\
\text { methods }\end{array}$} \\
\cline { 3 - 6 } & & RPA[1/1] & RPA[0/1] & PA[1/1] & PA[0/1] \\
\hline 0.1 & 500 & $4.30 \times 10^{-16}$ & $2.44 \times 10^{-15}$ & $2.73 \times 10^{-3}$ & $7.38 \times 10^{-2}$ \\
0.5 & & $4.44 \times 10^{-16}$ & $2.38 \times 10^{-15}$ & $3.73 \times 10^{-3}$ & $7.38 \times 10^{-2}$ \\
0.9 & & $2.77 \times 10^{-16}$ & $2.38 \times 10^{-15}$ & $5.32 \times 10^{-3}$ & $7.38 \times 10^{-2}$ \\
\hline 0.1 & 1000 & $1.92 \times 10^{-15}$ & $2.44 \times 10^{-15}$ & $4.55 \times 10^{-3}$ & $7.38 \times 10^{-2}$ \\
0.5 & & $1.83 \times 10^{-15}$ & $2.38 \times 10^{-15}$ & $4.04 \times 10^{-3}$ & $7.38 \times 10^{-2}$ \\
0.9 & & $1.87 \times 10^{-15}$ & $2.38 \times 10^{-15}$ & $2.93 \times 10^{-3}$ & $7.38 \times 10^{-2}$ \\
\hline 0.1 & 1500 & $3.63 \times 10^{-15}$ & $2.44 \times 10^{-15}$ & $3.98 \times 10^{-3}$ & $7.38 \times 10^{-2}$ \\
0.5 & & $3.66 \times 10^{-15}$ & $2.38 \times 10^{-15}$ & $3.70 \times 10^{-3}$ & $7.38 \times 10^{-2}$ \\
0.9 & & $3.44 \times 10^{-15}$ & $2.38 \times 10^{-15}$ & $4.40 \times 10^{-3}$ & $7.38 \times 10^{-2}$ \\
\hline 0.1 & 2000 & $4.19 \times 10^{-15}$ & $2.44 \times 10^{-15}$ & $2.79 \times 10^{-3}$ & $7.38 \times 10^{-2}$ \\
0.5 & & $4.27 \times 10^{-15}$ & $2.38 \times 10^{-15}$ & $4.07 \times 10^{-3}$ & $7.38 \times 10^{-2}$ \\
0.9 & & $4.48 \times 10^{-15}$ & $2.38 \times 10^{-15}$ & $4.72 \times 10^{-3}$ & $7.38 \times 10^{-2}$ \\
\hline
\end{tabular}


Table (2)

Comparison of the absolute errors (A.E.) between the considered methods RPA[1/1], RPA[0/1] and The classical methods PA[1/1], PA[0/1], for $h=0.1, k=1 \times 10^{-5}$ and $\delta=1 \times 10^{-3}$ for case $\mathrm{l}$.

\begin{tabular}{|c|c|c|c|c|c|}
\hline$x$ & $\begin{array}{c}\text { No. of } \\
\text { Steps }\end{array}$ & \multicolumn{2}{|c|}{$\begin{array}{c}\text { A. E. of The considered } \\
\text { methods }\end{array}$} & \multicolumn{2}{c|}{$\begin{array}{c}\text { A. E. of The classical } \\
\text { methods }\end{array}$} \\
\cline { 3 - 7 } & & RPA[1/1] & RPA[O/1] & PA[1/1] & PA[0/1] \\
\hline 0.1 & 500 & $8.68 \times 10^{-15}$ & $1.35 \times 10^{-14}$ & $1.78 \times 10^{-3}$ & $4.03 \times 10^{-3}$ \\
0.5 & & $9.82 \times 10^{-15}$ & $1.37 \times 10^{-14}$ & $2.81 \times 10^{-3}$ & $5.60 \times 10^{-3}$ \\
0.9 & & $1.12 \times 10^{-14}$ & $1.51 \times 10^{-14}$ & $3.91 \times 10^{-3}$ & $7.25 \times 10^{-3}$ \\
\hline 0.1 & 1000 & $1.21 \times 10^{-14}$ & $1.07 \times 10^{-15}$ & $4.03 \times 10^{-3}$ & $6.99 \times 10^{-3}$ \\
0.5 & & $1.01 \times 10^{-14}$ & $8.52 \times 10^{-15}$ & $2.73 \times 10^{-3}$ & $5.63 \times 10^{-3}$ \\
0.9 & & $8.99 \times 10^{-15}$ & $7.97 \times 10^{-15}$ & $1.82 \times 10^{-3}$ & $4.37 \times 10^{-3}$ \\
\hline 0.1 & 1500 & $8.54 \times 10^{-15}$ & $2.94 \times 10^{-15}$ & $1.69 \times 10^{-3}$ & $4.62 \times 10^{-3}$ \\
0.5 & & $1.06 \times 10^{-14}$ & $4.19 \times 10^{-15}$ & $2.96 \times 10^{-3}$ & $5.67 \times 10^{-3}$ \\
0.9 & & $1.03 \times 10^{-14}$ & $4.12 \times 10^{-15}$ & $1.88 \times 10^{-3}$ & $6.66 \times 10^{-3}$ \\
\hline 0.1 & 2000 & $1.11 \times 10^{-15}$ & $3.60 \times 10^{-16}$ & $3.81 \times 10^{-3}$ & $6.39 \times 10^{-3}$ \\
0.5 & & $9.90 \times 10^{-15}$ & $1.60 \times 10^{-15}$ & $2.84 \times 10^{-3}$ & $5.65 \times 10^{-3}$ \\
0.9 & & $9.02 \times 10^{-15}$ & $2.51 \times 10^{-15}$ & $1.88 \times 10^{-3}$ & $4.92 \times 10^{-3}$ \\
\hline
\end{tabular}

Table (3)

Comparison of the absolute errors (A.E.) between the considered methods RPA[1/1], RPA[0/1] and The classical methods PA[1/1], PA[0/1], for $h=0.1, k=1 \times 10^{-8}$ and $\delta=1 \times 10^{-4}$

\begin{tabular}{|c|c|c|c|c|c|c|c|}
\hline \multirow[t]{3}{*}{$x$} & \multirow{3}{*}{$\begin{array}{c}\text { No. } \\
\text { of } \\
\text { Steps }\end{array}$} & \multicolumn{4}{|c|}{ A. E. of The considered methods } & \multicolumn{2}{|c|}{$\begin{array}{l}\text { A. E. of The classical } \\
\text { methods }\end{array}$} \\
\hline & & \multicolumn{2}{|c|}{ Case I } & \multicolumn{2}{|c|}{ Case II } & \multirow[t]{2}{*}{$\mathrm{PA}[1 / 1]$} & \multirow[t]{2}{*}{$\mathrm{PA}[0 / 1]$} \\
\hline & & RPA[1/1] & $\mathrm{RPA}[0 / 1]$ & RPA[1/1] & RPA[O/1] & & \\
\hline 0.1 & 500 & $2.7 \times 10^{-14}$ & $4.2 \times 10^{-14}$ & $7.7 \times 10^{-7}$ & $7.7 \times 10^{-7}$ & $7.7 \times 10^{-5}$ & $7.5 \times 10^{-5}$ \\
\hline 0.5 & & $3.5 \times 10^{-14}$ & $4.6 \times 10^{-14}$ & $1.3 \times 10^{-6}$ & $1.3 \times 10^{-6}$ & $1.3 \times 10^{-4}$ & $1.2 \times 10^{-4}$ \\
\hline 0.9 & & $2.7 \times 10^{-14}$ & $1.1 \times 10^{-13}$ & $2.2 \times 10^{-6}$ & $2.2 \times 10^{-6}$ & $2.2 \times 10^{-4}$ & $2.1 \times 10^{-4}$ \\
\hline 0.1 & 1000 & $9.4 \times 10^{-14}$ & $6.5 \times 10^{-14}$ & $1.2 \times 10^{-6}$ & $1.2 \times 10^{-6}$ & $1.2 \times 10^{-4}$ & $1.2 \times 10^{-4}$ \\
\hline 0.5 & & $6.7 \times 10^{-14}$ & $1.2 \times 10^{-13}$ & $2.4 \times 10^{-6}$ & $2.4 \times 10^{-6}$ & $2.4 \times 10^{-4}$ & $2.4 \times 10^{-4}$ \\
\hline 0.9 & & $2.4 \times 10^{-14}$ & $3.2 \times 10^{-13}$ & $4.7 \times 10^{-6}$ & $4.7 \times 10^{-6}$ & $4.7 \times 10^{-4}$ & $4.6 \times 10^{-4}$ \\
\hline 0.1 & 1500 & $10^{-14}$ & $7.4 \times 10^{-14}$ & $1.5 \times 10^{-6}$ & $1.5 \times 10^{-6}$ & $1.5 \times 10^{-4}$ & $1.5 \times 10^{-4}$ \\
\hline 0.5 & & $8.3 \times 10^{-14}$ & $2.0 \times 10^{-13}$ & $3.5 \times 10^{-6}$ & $3.5 \times 10^{-6}$ & $3.5 \times 10^{-4}$ & $3.4 \times 10^{-4}$ \\
\hline 0.9 & & $1.3 \times 10^{-13}$ & $5.9 \times 10^{-13}$ & $7.4 \times 10^{-6}$ & $7.4 \times 10^{-6}$ & $7.4 \times 10^{-4}$ & $7.2 \times 10^{-4}$ \\
\hline 0.1 & 2000 & $6.8 \times 10^{-14}$ & $6.9 \times 10^{-14}$ & $1.6 \times 10^{-6}$ & $1.6 \times 10^{-6}$ & $1.6 \times 10^{-4}$ & $1.6 \times 10^{-4}$ \\
\hline 0.5 & & $8.1 \times 10^{-14}$ & $2.8 \times 10^{-13}$ & $4.5 \times 10^{-6}$ & $4.5 \times 10^{-6}$ & $4.5 \times 10^{-4}$ & $4.3 \times 10^{-4}$ \\
\hline 0.9 & & $3.0 \times 10^{-13}$ & $9.2 \times 10^{-13}$ & $1.0 \times 10^{-5}$ & $1.0 \times 10^{-5}$ & $1.0 \times 10^{-3}$ & $1.0 \times 10^{-3}$ \\
\hline
\end{tabular}

\section{Conclusion}

The numerical results presented in case $\mathrm{I}$ in each of tables (1), (2) and (3) shows that the absolute errors obtained by the considered methods is almost of order $10^{-10}$ of that absolute errors obtained by the classical methods. Also, the numerical results presented in case II in table (3) shows that the best absolute errors estimation for the classical methods is not better than $10^{-5}$, while for the 
considered methods the maximum absolute errors estimation dose not exceed that $10^{-5}$.

\section{References}

[1] Hassan N. A. Ismail and Elsayed M. E. Elbarbary "Restrictive Pade Approximation " Journal of Faculty of Education, Ain Shams University. Special Issue for First National Conference of Mathematical and Physical Science and Applications, pp. 63-76, Cairo,(1995).

[2] Hassan N. A. Ismail and Elsayed M. E. Elbarbary "Restrictive Pade" Approximation and Parabolic Partial Differential Equation "Int. J. Computer Math. Vol. 66, No. 34 pp. 343-351 (1998).

[3] Hassan N. A. Ismail and Elsayed M. E. M. Elbarbary " Highly Accurate Method for the Convection-Diffusion Equation "Accepted for Publications for Int. J. Computer Math. Vol. 72, pp. 271-280 (1999).

[4] Hassan N. A. Ismail " Accelerating Technique for Any Implicit Finite Difference Schemes for Two Dimensional Initial Boundary Value Problem for Parabolic Partial Differential Equations " $8^{\text {th }}$ Conference on Applied Mechanics \& Mechanical Engineering, pp 547-554, MTC, Cairo (1998).

[5] Hassan N. A. Ismail Elsayed M.E.M.Elbarbary and Adel Younes Hassan " Highly Accurate Method for Solving Initial Boundary Value Problem for First Order Hyperbolic Differential Equations " $24^{\text {th }}$ Int. Conf. For Stat. \& Comp. Sc. And its Applications, pp. 77-91, Cairo, (1999). Accepted for Publication in Int. J. Computer Math Vol. 00. pp. 1-11 (2000).

[6] Hassan N. A. Ismail and Adel Younes Hassan "Restrictive Pade" Approximation for Solving First Order Hyperbolic Partial Differential Equations " J. of Institute of Math. \& Computer Sciences Vol. 11 No. 1, pp. 63-71 (2000).

[7] Hassan N. A. Ismail Elsayed M.E.M.Elbarbary and Adel Younes Hassan" Restrictive Pade' Approximation for Solving First Order Hyperbolic Systems in One Space Dimension "25 $5^{\text {th }}$ int. Conf. For Stat. \& Computer Sc. And Application, pp. 19-25, Cairo (2000). To Appear in Int.J.Computer Math (2000).

[8] Hassan N. A. Ismail and Adel Younes Hassan "Restrictive Pade' Approximation for Solving First Order Hyperbolic Partial Differential Equations in Two space Dimensions " To Appear in J. of Institute of Math. \& Computer Sciences (2000).

[9] Hassan N. A. Ismail and Adel Younes Hassan "Restrictive Pade' Approximation for Solving First Order Hyperbolic System in Two space Dimensions " Sent to Siam Journal on Scientific Computing (2000).

[10] Ibrahim M. A. K, Shamardan A. B. and Yousef Y.K. "On the Numerical Solution of Singularly Perturbed Parabolic Partial Differential Equations " J. of Natural Sciences and Mathematics, Vol. 27 No. 1 pp. 41-50 (1987),

[11] Smith G. D. "Numerical Solution of Partial Differential Equations: Finite Difference Methods" Clarendon Press Oxford (1985). 